\title{
Experimental evidence of micro-EHL lubrication in rough soft contacts
}

\author{
Michele Scaraggi \\ DIMeG, Politecnico di Bari, V.le Japigia 182, 70126 Bari, IT and \\ Department of Mechanical Engineering, \\ Imperial College London, South Kensington Campus, \\ Exhibition Road, SW7 2AZ London, UK \\ Giuseppe Carbone \\ DIMeG, Politecnico di Bari, V.le Japigia 182, 70126 Bari, IT \\ Daniele Dini \\ Department of Mechanical Engineering, \\ Imperial College London, South Kensington Campus, \\ Exhibition Road, SW' $2 A Z$ London, UK
}

\begin{abstract}
An experimental investigation of the lubricated steady sliding contact for elastically soft solids is reported. We show that the anisotropic character of the surface roughness has a strong influence in determining the transition from boundary lubrication, where the normal load is fully supported by the asperity-asperity interactions, to the hydrodynamic regime, where a thin fluid layer completely separates solid surfaces from direct contact. In particular, tests have been carried out using a ball-on-flat test configuration to measure the friction coefficient at the contact between a smooth steel ball and a rough PDMS as a function of the sliding speed. The most noteworthy result is that the presence of roughness anisotropy strongly modifies the classical shape of the Stribeck curve as a consequence of local micro-EHL conditions which occur at the contact interface.
\end{abstract}




\section{INTRODUCTION}

Compliant contacts, most commonly known as soft contacts, are quite common in nature (e.g. sinovial joint lubrication, eye-eyelid contact, human skin contact) and technology (e.g. tyres, rubber sealings, wiperblades) [1]-[4]. A very classical example is within human body applications, e.g. food oral processing and tactile perception. The latter is the result of the stimuli experienced from soft contacts with the environment and since the sensory evaluation is one of the most important factor in the decision-making process of consumers, soft tribology is becoming highly relevant for the consumer industry [5]-[8]. In machine elements applications instead (as in the case of sealings [9], [10]), controlling the friction, leakage and wear properties is crucial for the safe operation of such contacts.

Roughness, among other physical quantities, has been shown to play a key role in the friction and adhesive characteristics of soft contacts [11]. Indeed, in bio-mimetics research it is actually widely recognized that a correctly designed hierarchical surface structuring can allow to efficiently obtain tailored tribological properties. This is the case of the Geckos adhesive-like research. Geckos (Gekko gecko) and many insects show an extraordinary climbing ability on vertical surfaces and ceilings, and this mechanism of biological attachment is ascribed to the co-working characteristics of roughness hierarchy, fluid secretion (capillarity and viscosity effects) and van der Waals interaction [12]-[14]. However, in lubricated soft contacts the combined effect of roughness and surface energies has been only recently investigated [7]. In Ref. [7] the authors performed ball-on-disk friction measurements on randomly rough rubber samples. They clearly showed that, in the case of hydrophobic conjunctions, reducing the roughness magnitude determines, beside the expected increase of the boundary friction value, the reduction of the velocity value corresponding to the absolute minimum of the Stribeck friction curve as well as the reduction the same friction magnitude. For the hydrophilic conjunctions instead a full boundary regime was shown not to occur at all [7], due to negligible dewetting transitions at the interface [15], [16]. On the theoretical side, an investigation of lubricated soft contacts has been only recently proposed in Ref. [16], [17] and in Refs. [18], [19], where a novel mean field theoretical model has been presented to describe mixed lubrication conditions.

However, to the best of authors' knowledge, the role played by the roughness anisotropy on the solid contact and lubricant dynamics at the interface of soft contacts has not been 


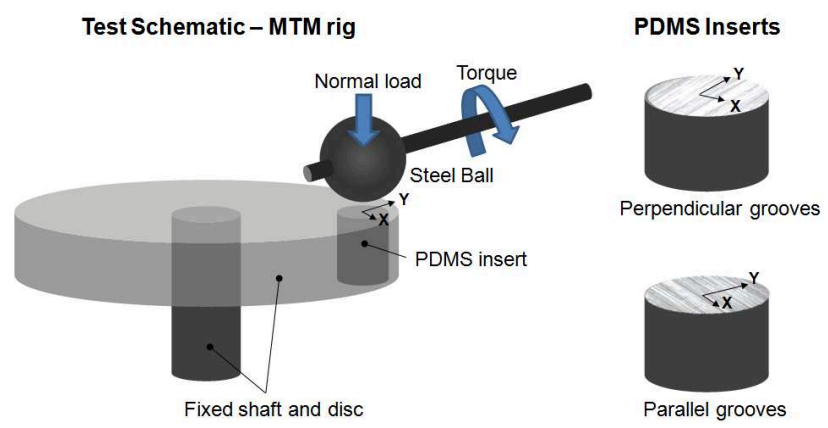

FIG. 1: Schematic of the contact geometry and measurement apparatus.

investigated yet. In this paper we focus on the experimental investigation of the effects of roughness anisotropy on the soft mixed lubrication and make use of recent theoretical and numerical developments [18]-[24] to, at least qualitatively, explain the behavior observed in our experimental investigations. Our setup consists of a metal ball in a steady-sliding contact with a fixed (anisotropically) rough PDMS substrate, see Fig. 1. We show that depending on the roughness orientation, very different friction curves can be measured; in particular, two distinct EHL regimes can be identified when, due to the specific orientation of the micro-features constituting the roughness, a full micro-EHL regime occurs in a velocity range not overlapping the macroscopic (ball-on-flat) EHL velocity range.

\section{THE EFFECT OF MICRO-EHL CONTACTS ON THE MACROSCOPIC CON- TACT FRICTIONAL BEHAVIOR}

We have carried out experiments by measuring friction force on a mini-traction machine (MTM), a tribometer designed to detect friction at sufficiently low loads as those encountered in soft lubrication conditions [8]. The contact geometry is very simple: a smooth (mirror finished) metal ball is rotated and loaded against a fixed rough elastomeric (macroscopically) flat substrate, as shown in Fig. 1. The contact is immersed in a lubricant bath and the temperature is monitored throughout the test. The ball is a stainless steel ball (AISI 440, radius $R=12.7 \mathrm{~mm}$ ). The elastomer surfaces are made of PDMS, fabricated from a twocomponent silicone elastomer kit (Sylgard 184, Dow Corning). We used a base and curing agent in a mass ratio of $10: 1$. The PDMS samples were made by casting a $5 \mathrm{~mm}$ thick sheet in between two aluminium plates, one of which has been roughened as described in the 
following paragraph, then the PDMS was cured for $45^{\prime}$ in the oven at $150{ }^{\circ} \mathrm{C}$. After cooling, the PDMS sheet was removed from the aluminium plates and $10 \mathrm{~mm}$ radius circular samples were cut from the sheet using a punching tool. The Young's modulus of the PDMS was observed to be about 2.3 $\mathrm{MPa}$, and the reduced elastic modulus for the elastomer-steel ball contact was found to be $E^{*}=3 M P a$, in line with the data provided by [10]. The applied load was $F=1 \mathrm{~N}$, with a calculated Hertz contact radius $a=1.46 \mathrm{~mm}$ and a maximum Hertz contact pressure $p_{\max }=0.22 \mathrm{MPa}$. However, we note that FEM calculations have shown that a slightly larger maximum contact pressure is expected, due to the finite thickness of the rubber sheet and to the proximity of metallic supporting substrate.

In order to generate roughness on the PDMS substrate, a flat aluminium plate has been initially sandblasted, thus producing an isotropic roughness with $\mathrm{rms}\left\langle h^{2}\right\rangle^{1 / 2} \approx 10 \mu \mathrm{m}$. A further roughening of the sample was obtained on the lethe under the action of a rotating rod covered with sand paper P60. This latter action did not significantly changed the magnitude of the rms of the surface, but generated a strong anisotropic rough surface, of which we show in Fig. 2 an image obtained with optical microscopy. In Fig. 3-a we also show a surface roughness measurement of the molding sheet, obtained using white light interferometry. In Fig. 3-b two 1D cross-sections of the roughness surface are shown. The two cross sections correspond to the location of the two (blue and red) curves in Fig. 3-a, which have been taken along two orthogonal directions. The difference between the two curves shows that the surface is actually anisotropic. The lubricants utilized during the experiments have been obtained by mixing at different concentrations the AnalaR NORMAPUR Glycerol bidistilled (code 24388.320) and distilled water (in order to obtain different lubricant viscosities $\eta$ ), see Tab. I. Due to the low molecular weight of the mixture, a Newtonian behavior is kept till shear strain rate value of about $10^{4} \mathrm{~s}^{-1}$, see e.g. [25]. Tests were carried out in simple sliding condition, obtained with the metallic ball rotating on the fixed PDMS sample, in order to avoid any (although unlikely) visco-elastic friction contribution from the rubber. Friction was measured over a sliding speed $U$ range of 0.004 to $0.5 \mathrm{~m} / \mathrm{s}$. The test protocol was as follows. Each single $\mu-\eta U$ curve is obtained by using only one rubber sample and different glycerine in water solutions. Moreover, each $(\eta U, \mu)$ point was calculated by averaging $5+5$ consecutive measurements (with temperature corrected viscosity): five experiments have been carried out with sliding velocity $U$ and the other five with sliding velocity $-U$, in order to remove possible bias error of the load cell. Measurements have also been performed in 


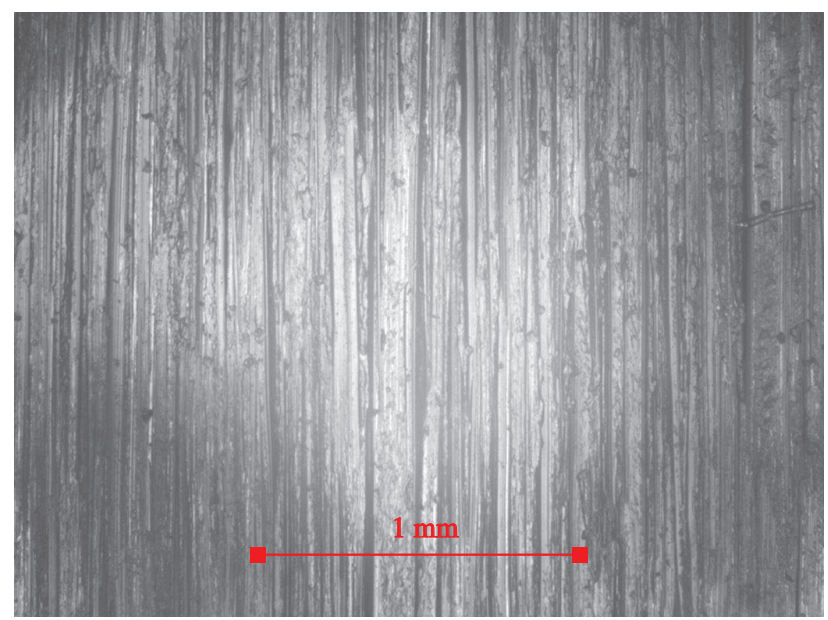

FIG. 2: Optic microscope acquisition of a surface roughness similar to the ones we used in the experiments.

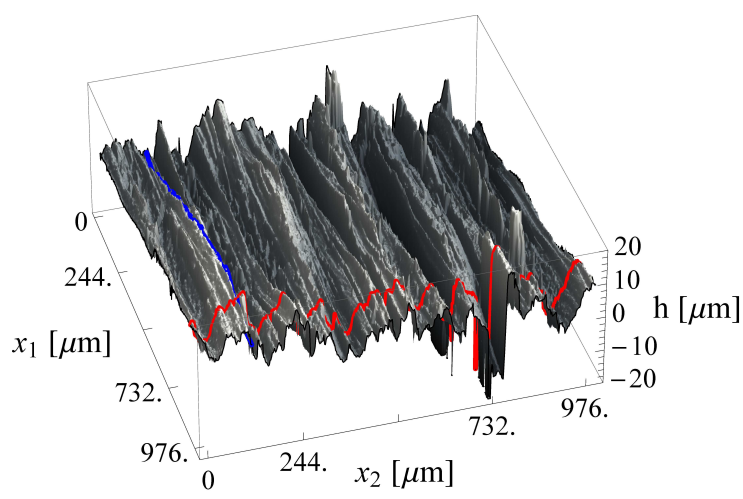

(a)Three-dimensional roughness map

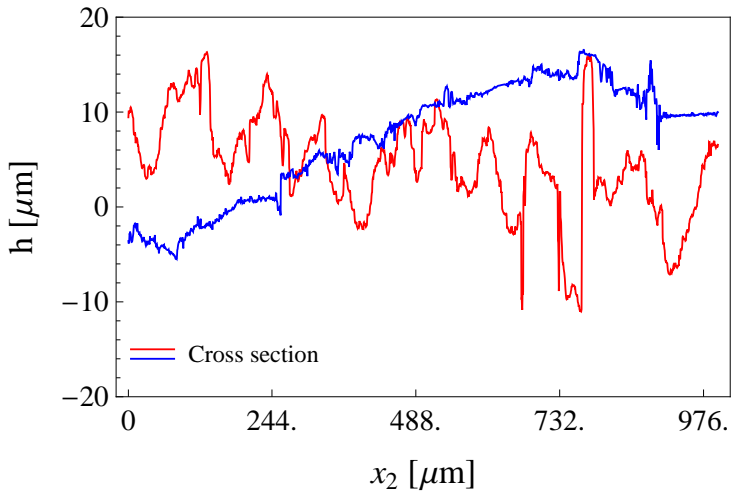

(b)Cross-sections

FIG. 3: A sample of anisotropic surface roughness measured on the molding plate.

\begin{tabular}{|c|c|}
\hline \multicolumn{2}{|c|}{ Dynamic viscosity of glycerine $\%_{w}$ in water } \\
$\%_{w}$ & $\eta$ at $20^{\circ} \mathrm{C}[\mathrm{Pa} \mathrm{s}]$ \\
\hline \hline 56 & 0.0086 \\
79 & 0.053 \\
92 & 0.31 \\
98 & 0.94 \\
\hline
\end{tabular}

TABLE I: Lubricants viscosities employed in this study at room temperature. 


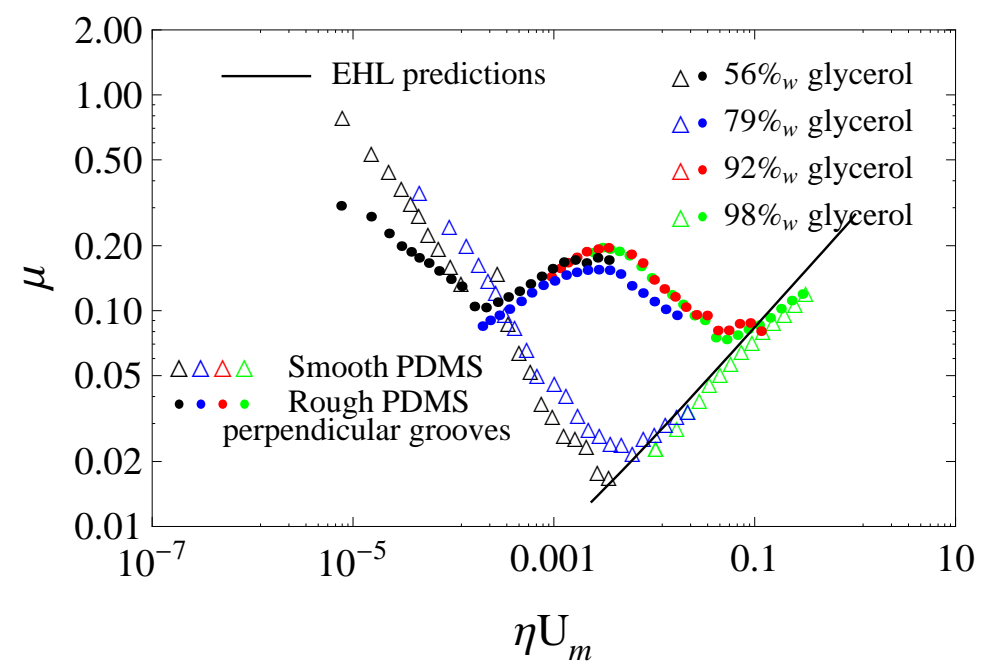

FIG. 4: Friction coefficient $\mu$ as function of the product dynamic viscosity $\mu$ and mean velocity $U_{m}=U / 2$ (curve $\mu-\eta U$ ), for the rough surface with grooves perpendicular to the sliding direction, and for the smooth rubber sample.

a random order to confirm measurements repeatability. Friction measurements were also carried out in pure water, however in this particular test configuration the hydrophobic nature of the PDMS caused the lubricant to be completely squeezed out from the interface as the operating conditions approached the boundary lubrication regime. This resulted in strong wear of the rubber surface (clearly visible using optical microscopy) and consequent loss of measurement repeatability. Therefore, these results are not presented in this paper. After each set of measurements with the same lubricant, all those parts of the apparatus in direct contact with the lubricant have been washed with a copious amount of distilled water, followed by alternating use of isopropanol and acetone. Mobile parts have been also washed by 15-minutes ultrasonic bath cleaning in isopropanol.

In Fig. 4 we show the measured friction curves, whereas in Fig. 5 we report the calculation results [16] of the minimum film thickness for the elasto-hydrodynamic case in the same contact conditions adopted in the experiments. Measurements are reported for both the smooth PDMS sample $\left(\left\langle h^{2}\right\rangle^{1 / 2} \approx 0.1 \mu m\right)$ and the rough PDMS sample with roughness anisotropy characterised by surface "grooves" aligned in a direction perpendicular to the ball sliding velocity. The smooth PDMS has been prepared by casting against an aluminium sheet not sandblasted, characterized by a very small roughness compared to the sandblasted surface. 


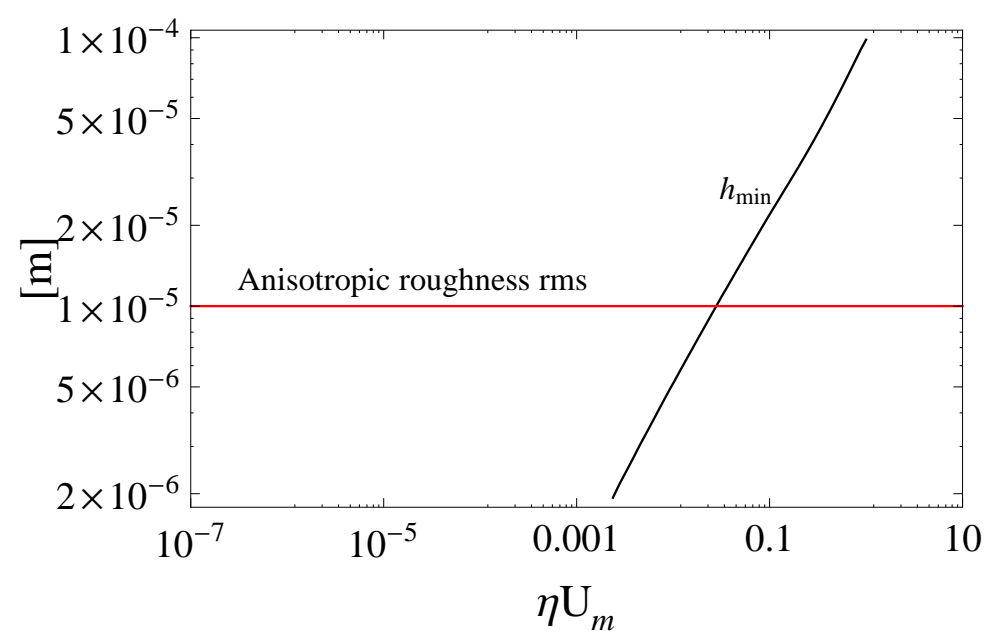

FIG. 5: Minimum film thickness as function of the product $\eta U_{m}$ (black curve) [16]. For the elastohydrodynamic case and in the same contact conditions adopted in the experiments. The red line qualitatively corresponds to the anisotropic roughness rms.

Interestingly Fig. 4 shows the appearance of two hydrodynamic regimes in the friction curve of the rough surface. The first, which represents the most important results of our investigation and has been never observed before, initiates at about $\eta U_{m}=10^{-4} \mathrm{~Pa} \mathrm{~m}$, the second at about $\mu U_{m}=0.1 \mathrm{~Pa} m$ and, as expected, rapidly follows the classical EHL Stribeck curve for smooth bodies. The former transition is (to the best of authors knowledge) the very first experimental observation of friction modification induced by anisotropic roughness in soft micro-EHL lubrication [1]. It occurs in a velocity range where the macroscopic EHL contribution coming from the ball curvature radius is still negligible and cannot explain the observed friction behavior. We observe that micro-EHL occurs locally at the individual asperities, each of them being characterised by a local hydrodynamic regime. Indeed, if the sliding velocity is increased, also the average interfacial separation increases. This in turn diminishes the pressure fluctuations caused by the fluid-asperity interactions and the microEHL phenomenon disappears, being replaced by the hydrodynamic regime determined by the ball macroscopic shape. We stress that one of the reasons of the occurrence of this large friction increase is a consequence of the large separation of length scales between the length of the macroscopic contact (at which macro-EHL occurs) and the largest wavelengths of the surface roughness (where micro-EHL occurs). Under the velocity range where micro-EHL 


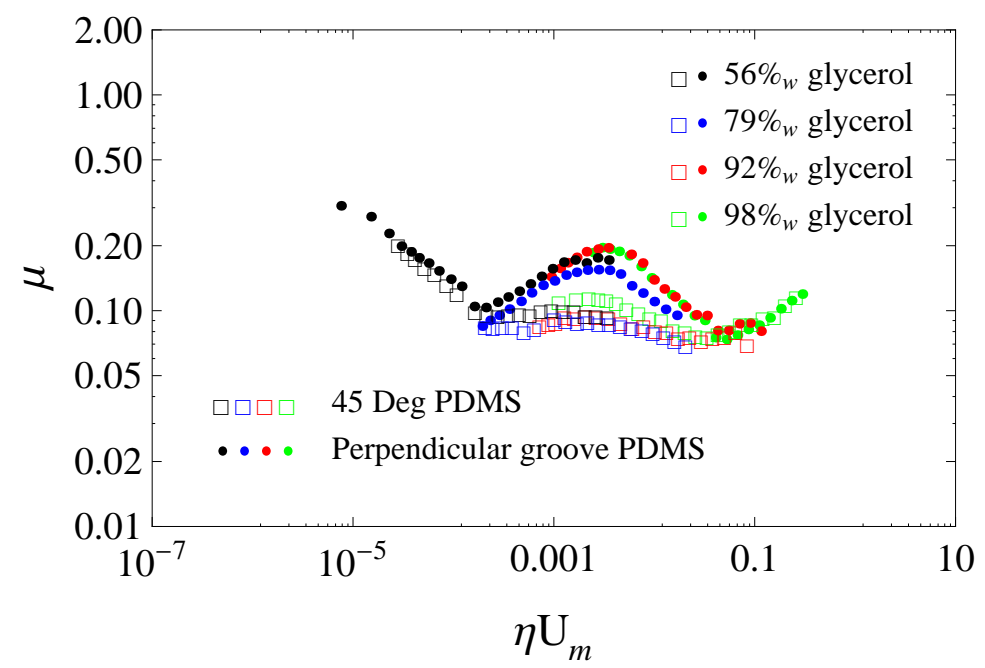

FIG. 6: Friction coefficient $\mu$ as function of the product dynamic viscosity $\mu$ and mean velocity $U_{m}=U / 2$ (curve $\mu-\eta U$ ), for the rough surface with grooves perpendicular to the sliding direction, and for the same surface but with grooves 45-degrees aligned to the sliding direction.

appears, the contact is almost conformal at the macroscopic level, however this is not necessarily true at the micro-scale. Local asperities will generate local non-conformal contacts which lead to the establishment of local micro-EHL conditions, which strongly depends on complex fluid-solid interactions. We also observe that the local maximum of the friction curve for the case of rough PDMS corresponds to the position of the lowest friction value measured in the case of the smooth surface, i.e. at the onset of macroscopic hydrodynamic behaviour governed by the ball geometry. This result suggests that micro-EHL conditions would still govern the frictional behavior of the interface even at larger velocities provided that macro-EHL never takes place. However, as soon as the latter occurs, the micro-EHL effect should necessarily be reduced or disappear.

Figure 6 shows the results obtained when the PDMS insert is rotated to make the grooves of the rough surface tilted of $45^{\circ}$ with respect to the sliding velocity. As expected, the variation in friction induced by the $45^{\circ}$ tilted configuration is lower than that obtained in the case of perpendicularly oriented grooves. A possible reason for such a behavior has been recently proposed in Refs. [18] and [19], where the authors showed that, at least in terms of asperities flattening, the strength of the fluid-asperity interactions is maximum for the transverse roughness, i.e. when the roughness grooves are aligned perpendicularly to the 


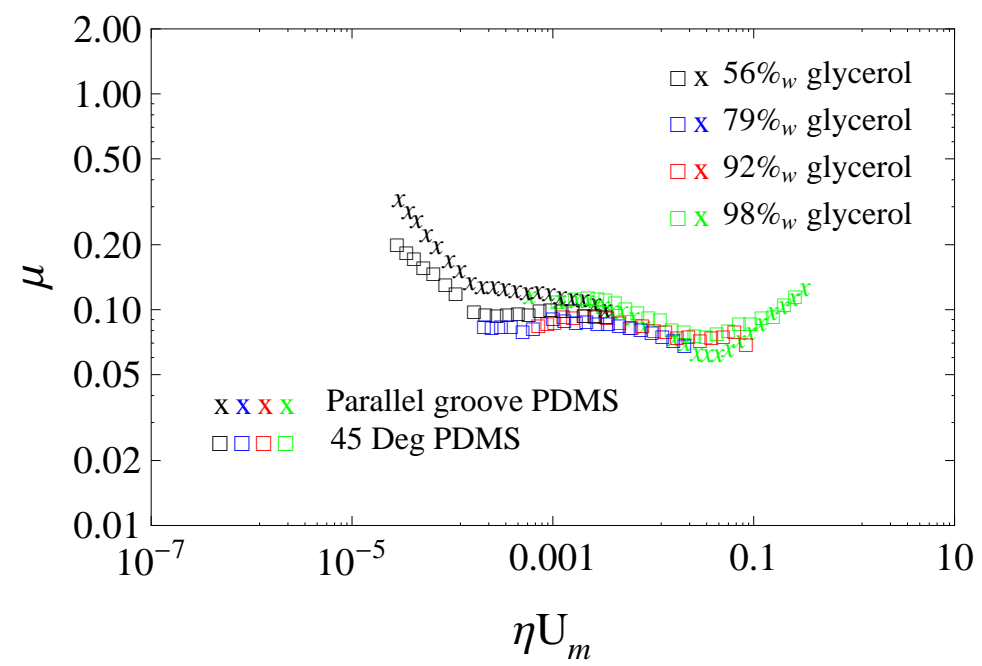

FIG. 7: Friction coefficient $\mu$ as function of the product dynamic viscosity $\mu$ and mean velocity $U_{m}=U / 2$ (curve $\mu-\eta U$ ), for the rough surface with grooves parallel to the sliding direction, and for the same surface but with grooves 45-degrees aligned to the sliding direction.

sliding direction (see also [20]-[24]). Asperity flattening is caused by the fluid overpressure generated by the surface roughness. Hence, larger asperity flattening and therefore stronger micro-EHL conditions should occur along those sliding directions where the induced fluid overpressure takes its maximum value. A very rough estimation of the fluid overpressure $\Delta p$ generated by a one-dimensional roughness of wavelength $\lambda$ and amplitude $h$ can be obtained by perturbing the one-dimensional Reynolds equation in the film thickness. Therefore assuming that the fluid overpressure $\Delta p$ is smaller that the pressure $\Delta p^{*} \approx E h / \lambda$ needed to completely flatten the one-dimensional roughness, it is easy to show that

$$
\Delta p \approx \frac{\mu U \lambda h \cos \theta}{u^{3}}
$$

where $u$ is the average separation, $\mu$ the fluid viscosity, $U$ the sliding velocity, and $\theta$ is the angle between the velocity and the roughness direction (zero for transverse roughness). Eq. (1) clearly shows that the maximum fluid overpressure is obtained for transverse roughness (i.e. for $\theta=0$ ).

This also suggests that full micro-EHL contacts are likely not to be present in the configurations at $45^{\circ}$ and $90^{\circ}$, where instead a similar mixed lubrication condition should be expected. This is confirmed in Fig. 7, where we compare the results obtained for the $45^{\circ}$ 
tilted grooves and for grooves aligned with the sliding velocity.

It should also be noted that, as suggested by one of the anonymous referees, the observed behaviour (the hat in Fig. 4) might be associated to the occurrence of starvation at asperity level. However, the authors believe that "micro-starvation" is unlikely to be responsible alone for the hydrodynamic behaviour observed experimentally for perpendicularly oriented grooves as the slope of the friction curve clearly suggests that an EHL-like mechanism takes place. Infact, a "starved micro-EHL" regime might be the only alternative to explain the slope of the curve obtained experimentally for the transverse roughness, therefore we suggest that a double hydrodynamic regime (micro- and macro-) would have been difficult to achieve in the absence of the micro-EHL mechanism. It is concluded that, in order to shed light on the interplay of the mechanisms governing the frictional behaviour observed in the tests presented in the present contribution, the behaviour at the contact interfaces should be carefully monitored and the local film thickness should be measured with very high resolution; this would enable capturing the behaviour of the fluid and the fluid/solid interactions at asperity level. However, such very demanding task is outside the scope of the present contribution.

Now let us observe that the increase of friction in the micro-EHL regime (for the transverse roughness) occurs almost similarly as in classical (macro) EHL regime (see e.g. Fig. 4). This simply supports our interpretation that roughness determines local elastohydrodynamic lubrication conditions. In order to clarify this, consider the black curves shown in Fig. 8 which concerns the results of numerical calculations (based on the methodology outlined in [16]) of fully EHL contacts for different radii and load conditions. To carry out the calculations we used four different ball radii, namely $R_{1}=R, R_{2}=R_{1} / 10, R_{3}=R_{1} / 10^{2}$, and $R_{4}=3.94 R_{1} / 10^{3}$, where $R_{4}$ is representative of the length scale of the anisotropic roughness of the PDMS samples used in the experiments reported above (we note however that point contacts have been used with the only purpose to adopt a uniform scaling law in the following qualitative friction comparison). The load $F$ utilized to carry out the numerical calculations has been scaled with a square law, as the number of asperities is proportional to the surface area and therefore scales with a square law. Then the load corresponding to the above radii are respectively $F_{1}=1 N, F_{2}=F_{1} / 10^{2}, F_{3}=F_{1} / 10^{4}$, and $F_{4}=1.55 F_{1} / 10^{5}$, so that the total load is conserved and friction curves can be compared on a consistent basis. Note that, as expected, reducing the radius determines only a shift toward lower 


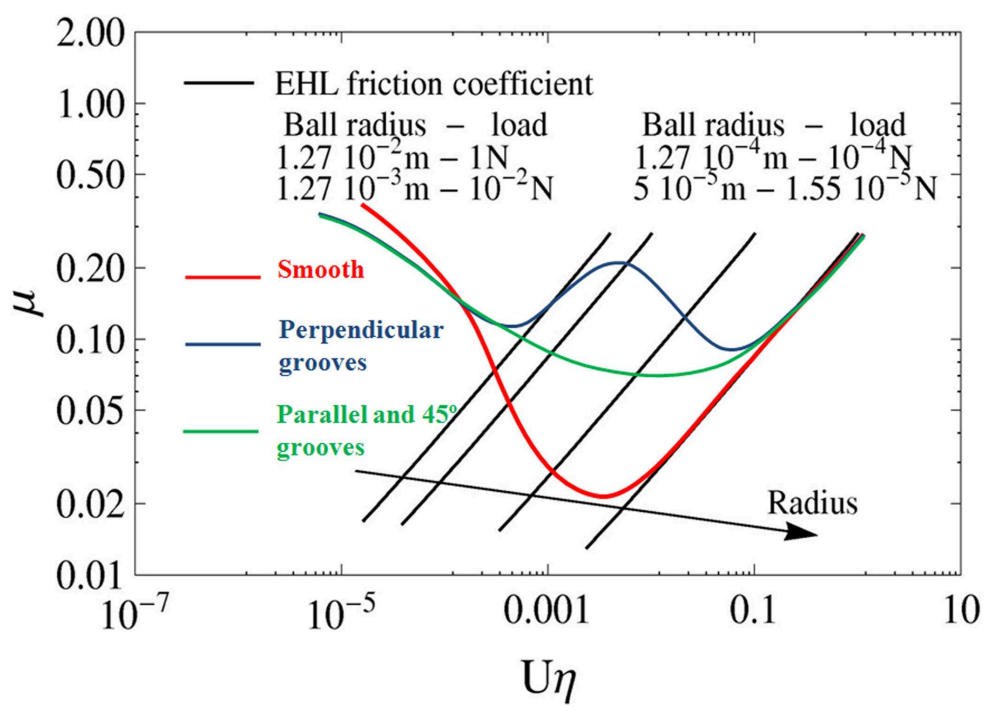

FIG. 8: The three experimental trends from Figs. 4 and 6 superimposed to four EHL predictions. Note the large enhancement of micro-EHL observed for the perpendicularly oriented grooves. The friction coefficient for pure EHL contacts has been calculated under the following conditions: ball radius (and corresponding load) $1.27 * 10^{-2} \mathrm{~m}(1 \mathrm{~N}), 1.27 * 10^{-3} \mathrm{~m}\left(10^{-2} \mathrm{~N}\right), 1.27 * 10^{-4} \mathrm{~m}\left(10^{-4} \mathrm{~N}\right)$ and $5.0 * 10^{-5} \mathrm{~m}\left(1.55 * 10^{-5} \mathrm{~N}\right)$, corresponding to the curves from right to left. The reduced elastic modulus is the same of the experimental setup.

values of the abscissa $\eta U_{m}$. Indeed, since the soft-EHL problem can be formulated in terms of two dimensionless parameter, namely the load parameter $\tilde{F}=F /\left(E^{*} R^{2}\right)$ and the speed parameter $\tilde{U}=U_{m} \eta /\left(E^{*} R\right)$, in our case we have $\tilde{F}_{1}=\tilde{F}_{2}=\tilde{F}_{3}=\tilde{F}_{4}$, so that velocities must scale with the radius to get the same speed parameter and therefore the same friction coefficient. This indicates that, with the same surface area but differently-sized micropatterns, similar friction forces can be produced in a broader velocity range at constant load condition. This is true as long as the micro-patterns govern the contact behaviour, which qualitatively explains our experimental results. In particular, the three experimental trends shown in Figs. 4 and 6 have been superimposed in Fig. 8 to the four EHL predictions: the plot seems to suggest that the micro-EHL observed for the perpendicularly oriented grooves takes place at the regime where the roughness length scales dominate the surface response and a full (local) EHL regime would be expected to develop at the velocity range which corresponds to the anisotropic roughness wavelength used in the experimental tests. 


\section{CONCLUSIONS}

We have performed friction measurements in the case of a smooth steel rotating ball in lubricated contact with a fixed rough PDMS counter surface. The rubber sample is characterized by a strongly anisotropic roughness. We report on the existence of microEHL lubrication states (for the transverse roughness configuration), which determine large differences in the measured friction curves if compared to the classical shape of the Stribeck curve.

\section{Acknowledgments}

MS and GC acknowledges Region Puglia for having supported the research activity through the constitution of the TRASFORMA Laboratory Network cod. 28. MS acknowledges the Tribology Group for the kind hospitality received during his visit to the Department of Mechanical Engineering at Imperial College London, where part of this work has been performed. MS also acknowledges Dr. C. Myant and Dr. M. Fowell for discussions and help in setting up the experimental set-up. GC acknowledges the support of the European Science Foundation EUROCORES - FANAS Programme - EC Sixth Framework Programme under contract N. ERASCT-2003-980409.

[1] Dowson, D.: Elastohydrodynamic and micro-elastohydrodynamic lubrication. Wear 190, 125138 (1995).

[2] Persson, B. N. J.: Sliding Friction: Physical Principles and Applications, Springer, 2nd edition (2000)

[3] Dowson, D.: Elastohydrodynamic Lubrication in 'Soft-On-Soft ' Natural Synovial Joints; ' Hard-On-Soft ' Cushion and 'Hard-On-Hard' Metal-On-Metal Total Joint Replacements. In Snidle, R. W. and Evans, H. P. (ed.), IUTAM Symposium on Elastohydrodynamics and Micro-elastohydrodynamics, Springer Netherlands, pp. 297-308 (2006)

[4] Scaraggi, M: Contact and friction modeling of rough surfaces in dry and lubricated contacts, PhD thesis, Politecnico di Bari (2010) 
[5] de Vicente, J., Stokes, J. R., Spikes, H. A.: The frictional properties of Newtonian fluids in rolling-sliding soft-EHL contact. Tribology Letters 20 (3-4), 273-286 (2005)

[6] de Vicente, J., Stokes, J. R., Spikes, H. A.: Soft lubrication of model hydrocolloids. Food Hydrocolloids 20, 483-491 (2006)

[7] Bongaerts, J. H. H., Fourtouni, K., Stokes, J. R.: Soft-tribology: Lubrication in a compliant PDMS-PDMS contact. Tribology International 40, 1531-1542 (2007)

[8] Myant, C., Spikes, H. A., Stokes, J. R.: Influence of load and elastic properties on the rolling and sliding friction of lubricated compliant contacts. Tribology International 43, 55-63 (2010)

[9] Shi, F., Salant, R. F.: A Mixed Soft Elastohydrodynamic Lubrication Model With Interasperity Cavitation and Surface Shear Deformation. Journal of Tribology 122, 308-316 (2000)

[10] Lorenz, B., Persson, B. N. J.: Leak rate of seals: Comparison of theory with experiment. EPL $86,44006(2009)$

[11] Persson, B. N. J.: Theory of rubber friction and contact mechanics. Journal of Chemical Physics 115 (8), 3840-3861 (2001)

[12] Gao, H., Wang, X., Yao, H., Gorb, S., Arzt, E.: Mechanics of hierarchical adhesion structures of geckos. Mechanics of Materials 37(2-3), 275-285 (2005)

[13] Zhang, H., Guo, D. J., Dai, Z. D.: Progress on gecko-inspired micro/nano-adhesion arrays. Chinese Science Bullettin 55(18), 1843-1850 (2010)

[14] Carbone, G., Pierro, E., Gorb, S.: Origin of the superior adhesive performance of mushroom shaped microstructured surfaces. Soft Matter, DOI:10.1039/C0SM01482F, (2011)

[15] Scaraggi, M., Carbone, G.: Transition from elastohydrodynamic to mixed lubrication in highly loaded squeeze contacts. Journal of the Mechanics and Physics of Solids 58 (9), 1361-1373 (2010)

[16] Persson, B. N. J., Scaraggi, M.: On the transition from boundary lubrication to hydrodynamic lubrication in soft contacts. J. Phys.: Condens. Matter 21 (18), 185002 (2009)

[17] Persson, B. N. J.: Fluid dynamics at the interface between contacting elastic solids with randomly rough surfaces. J. Phys.: Condens. Matter 22 (26), 265004 (2010)

[18] Scaraggi, M., Carbone, G., Persson, B. N. J., Dini, D.: Mixed lubrication in soft contacts: A novel homogenized approach. Part I - Theory. Submitted (2011)

[19] Scaraggi, M., Carbone, G., Dini, D.: Mixed lubrication in soft contacts: A novel homogenized approach. Part II - Discussion. Submitted (2011) 
[20] Hooke, C. J.: Surface roughness modification in elastohydrodynamic line contacts operating in the elastic piezoviscous regime. Proceedings of the Institution of Mechanical Engineers Part J-Journal of Engineering Tribology 212 (J2), 145-162 (1998)

[21] Hooke, C. J., Venner, C. H.: Surface roughness attenuation in line and point contacts. Proceedings of the Institution of Mechanical Engineers Part J-Journal of Engineering Tribology 214 (J5), 439-444 (2000).

[22] Hooke, C. J., Li, K. Y.: Rapid calculation of the pressures and clearances in rough, elastohydrodynamically lubricated contacts under pure rolling. Part 1: low amplitude, sinusoidal roughness. Proceedings of the Institution of Mechanical Engineers Part C-Journal Of Mechanical Engineering Science 220 (6), 901-913 (2006).

[23] Hooke, C. J., Li, K. Y.: Rapid calculation of the pressures and clearances in rough, elastohydrodynamically lubricated contacts under pure rolling. Part 2: general roughness. Proceedings of the Institution of Mechanical Engineers Part C-Journal Of Mechanical Engineering Science 220 (6), 915-925 (2006).

[24] Hooke, C. J.: Engineering analysis of rough elastohydrodynamically lubricated contacts. Proceedings of the Institution of Mechanical Engineers Part J-Journal of Engineering Tribology 223 (J3), 517-528 (1998).

[25] Dontula, P., Macosko, C. W., Scriven, L. E.: Does the Viscosity of Glycerin Fall at High Shear Rates?. Ind. Eng. Chem. Res 38, 1729-1735 (1999) 